\title{
GESTIÓN DEL CONOCIMIENTO Y CREATIVIDAD
}

Maité Darceles Tife*

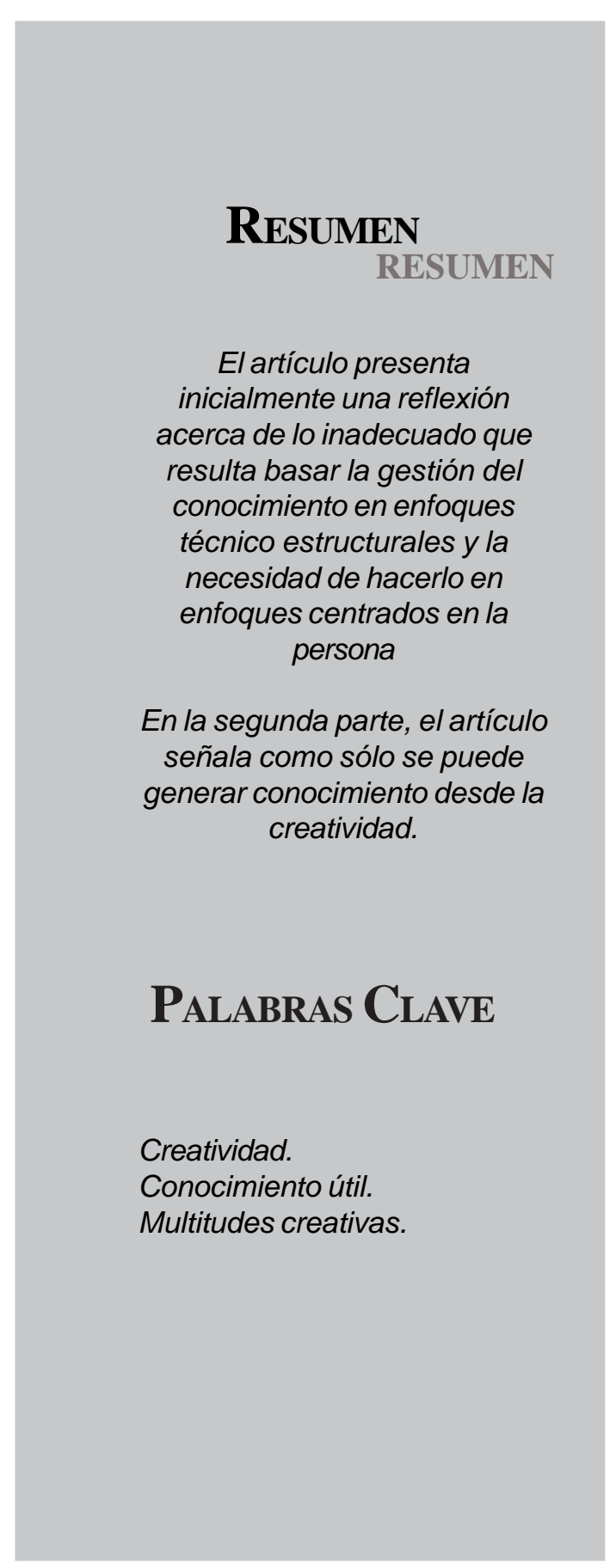

$\mathbf{E}$ el actual, el conocimiento y su gestión se convierten en claves competitivas de primer orden en las organizaciones. La gestión del conocimiento, es decir, cómo trata la organización el complejo campo del conocimiento, se suele abordar desde perspectivas bien distintas:

- El enfoque persona resalta la naturaleza del conocimiento como inmanente a la persona $y$, por tanto, se trata de propiciar ambientes que fomenten actitudes hacia comportamientos tendentes a generar y manejar conocimiento que resulte útil.

- El enfoque dato o información destaca las posibilidades de sistematización y procesamiento de la información como fuente de generación de mayor conocimiento. Las tecnologías de la información y la comunicación -llamadas TIC- en continuo desarrollo permiten manejar inmensas cantidades de información y procesar los datos en función de parámetros preestablecidos.

Recientemente leí un artículo $^{1}$ en el que se definían dos alternativas en la gestión del conocimiento: el enfoque del comportamiento y el enfoque técnico-estructural. Las autoras concluían que es necesario llegar a una integración de estos dos enfoques para optimizar la gestión del conocimiento.

La tesis del presente artículo es que el enfoque persona y el enfoque dato no se pueden

* Colaboradora del Cluster del Conocimiento. 
considerar enfoques alternativos del mismo nivel. El enfoque persona es básico, es donde radica el conocimiento, donde habita la creatividad, la innovación; mientras que el enfoque dato es un cúmulo de herramientas que pueden permitir manejar ingentes volúmenes de información incluso a tiempo real-, resultando un complemento del enfoque persona, y de ninguna manera un enfoque alternativo.

\section{La invalidez de enfoques técnico-estructurales}

El citado artículo me sirve para exponer mi punto de vista en parte discrepante, por lo que utilizaré citas literales del mismo, que resaltaré entre comillas.

«El enfoque del comportamiento aboga por la gestión de las condiciones relacionadas con el desarrollo del potencial humano como principal depositario del conocimiento. Desde esta perspectiva, los datos y la información no significan nada si no son correctamente interiorizados e interpretados por los miembros de la organización. En consecuencia, el progreso del conocimiento es un producto social que emerge de la interacción entre individuos e implica el desarrollo de procesos compartidos de interpretación de la realidad y las experiencias». Todo contraste con la realidad nos llevará a interpretaciones de este tipo.

«Desde el enfoque técnico-estructural, se proponen cuatro elementos de gestión del conocimiento como piezas básicas: la vigilancia del entorno, los procesos de planificación estratégica, los sistemas de información y los sistemas de evaluación y seguimiento". Las autoras proponían un equilibrio entre estos dos enfoques: «cualquiera de los dos enfoques, por separado, no está exento de limitaciones,

1 Elena Revilla e Isabel Prieto: «La dualidad de la gestión del conocimiento». Boletín Empresa Digitala, No.36, 2006.

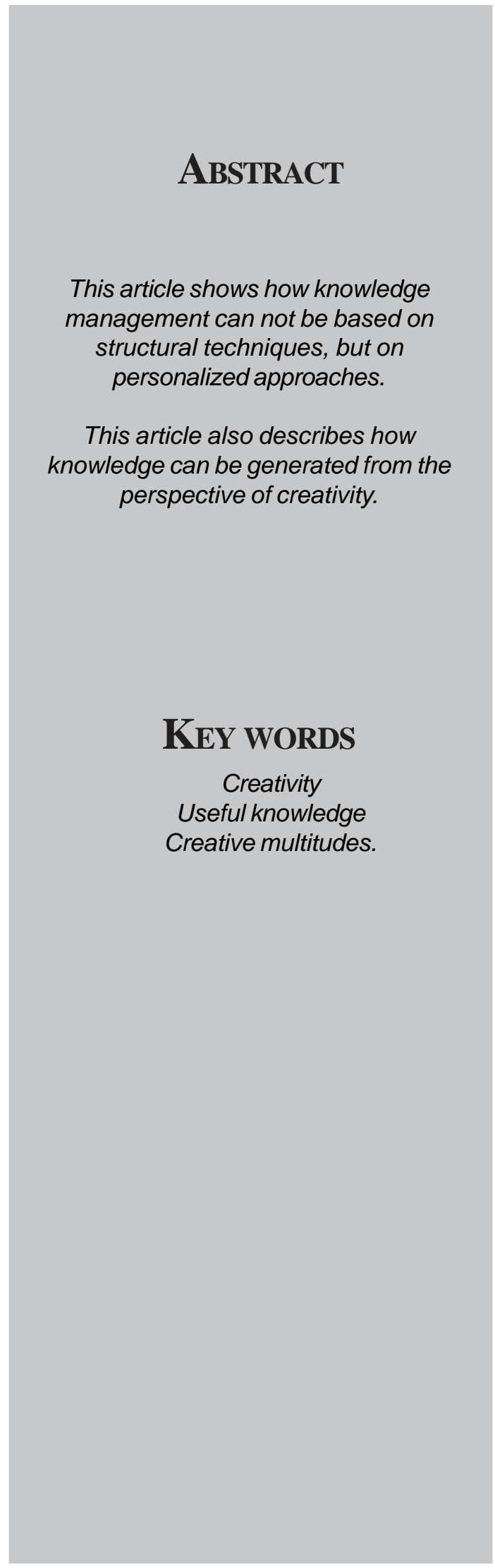


lo que ha propiciado la posterior evolución de estas dos propuestas hacia planteamientos integradores. De hecho, la reconciliación de ambas corrientes es necesaria a la hora de establecer un marco general de gestión del conocimiento. En definitiva, la gestión del conocimiento sólo es posible cuando existen valores compartidos e iniciativas orientadas al aprendizaje y al desarrollo del conocimiento que se complementan con unas estructuras, sistemas y procedimientos apropiados para hacerlos operativos».

La propuesta integradora de estas autoras adolecía de falta de profundización sobre las implicaciones de cada una de estas orientaciones, hasta el punto que los elementos que se proponían como piezas básicas de la gestión del conocimiento desde la perspectiva técnicoestructural, que pretendidamente debieran convivir armoniosamente con el enfoque del comportamiento, en la práctica, anularían -y de hecho en la gran mayoría de las organizaciones anulan- la generación de entornos positivos para un enfoque del comportamiento real. La idea de enfrentarnos a lo nuevo creando mayor conocimiento, se aleja intuitivamente de procedimientos, planes cerrados, decálogos y métodos. Dice Alfonso Vázquez que el concepto de los 'métodos de la innovación' es un oxímoron ${ }^{2}$. Se llega a lo nuevo a través de múltiples vías, y los métodos, que son por definición pasos conocidos, precisamente por esta razón, no son los caminos más fructíferos. Esta potente idea es extensible también a todo lo que guarda relación con la generación y manejo del conocimiento.

Un punto medio puede parecer la mejor solución desde el plano puramente teórico. Existen las perspectivas $A$ y $B$, que unimos en un cóctel perfecto, cogiendo lo mejor de cada una. Sin embargo, si profundizamos en su aplicación práctica, el pretendido equilibrio es imposible de lograr, porque cada una de las orientaciones implica un modelo distinto de despliegue de la estrategia. La organización selecciona su orientación principal en función de cómo ejecuta el proceso de definición estratégica. Este aspecto crucial es pasado por alto. Así, es más que probable que el proclamado equilibrio esconda una apuesta no consciente pero clara por la estrategia desplegada de forma vertical desde el enfoque técnico-estructural, que pretenderá maquillarse con planteamientos humanistas de poco alcance, con lo que la potencia del enfoque del comportamiento nunca llega a florecer.

Una organización basada en procedimientos planificados y cerrados como los citados desde el enfoque técnico-estructural subsume una estrategia desplegada verticalmente en sentido descendente. Mientras que el enfoque del comportamiento real requiere una forma de organización alternativa que permita la comunicación abierta, avanzar en la interpretación compartida de la realidad y el desarrollo de multitudes creativas, como origen de la estrategia.

Una vez defendida mi postura sobre la primacía del enfoque del comportamiento, voy a citar una vez más el artículo para apuntar que también las autoras se decantan entre líneas por este enfoque:

«Las organizaciones que quieran permanecer en un estado continuo de aprendizaje y mejora -en definitiva, sobrevivir- deberán moverse lejos de modelos pasivos en la gestión del conocimiento y embarcarse en los más inquietos».

Por supuesto, 'una gestión pasiva' [despreocupada hacia el conocimiento-] 'no genera el aprendizaje necesario en una situación de aumento de la inestabilidad'.

En este contexto, resulta que 'la organización [con orientación técnico-estructural] mantiene su capacidad para responder eficientemente a las situaciones que vayan surgiendo en el entorno, siempre que éstas se encuentren dentro del terreno de lo conocido'.

\footnotetext{
2 Figura retórica que consiste en la unión de dos palabras de significado opuesto.
} 
Esto significa que la única orientación en la gestión del conocimiento que prepara a la empresa para lo nuevo, para entornos inestables, es la que las autoras denominan la orientación del comportamiento. El enfoque técnicoestructural puede ser útil para tratar con condiciones constantes. Partiendo de variables inalteradas se pueden crear estructuras cada vez más perfectas que logren maximizar la eficiencia en la gestión del conocimiento que se genere. Ahora bien, pensar hoy en condiciones constantes es tan fantasioso que el enfoque no da demasiado juego.

La conclusión resulta clara: en los actuales entornos inestables y en continuo cambio el enfoque técnico-estructural tiene que estar supeditado al enfoque del comportamiento. Sin embargo, esta supeditación no es posible si el enfoque técnico-estructural se define como en el artículo, ya que los elementos que se definían como básicos no son compatibles con el enfoque del comportamiento llevado a la práctica. Por ello, tras estas explicaciones que me han servido de introducción, vuelvo a mi terminología, que me permite definir el enfoque persona y enfoque dato de manera compatible e interactuante.

Crear conocimiento consiste en llegar a una nueva relación entre conocimientos previos que abre nuevas vías de solución o exploración. Esta 'nueva relación' es el producto de la creatividad, de la imaginación. Sólo se puede generar conocimiento, imprescindible para innovar, desde la creatividad. Esta frase puede considerarse tautológica. Pero es que además, cuando el conocimiento llega a un nivel alto de complejidad, es imposible manejarlo inteligentemente sin poner en práctica la imaginación. Es obvio que generar nuevo conocimiento requiere creatividad, lo que no se oye tan a menudo -pero resulta igual de obvio a nada que pensemos sobre cualquier ejemplo práctico- es que optimizar su manejo también la requiere. Así, la gestión del conocimiento guarda estrechísima relación con el desarrollo de una multitud creativa.
En esta primera parte me he centrado en argumentar la ineficacia de basar la gestión del conocimiento en enfoques técnicoestructurales y la rotunda supremacía de enfoques persona. En su segunda parte completaré este artículo profundizando en el proceso de creación de conocimiento. Daré unas pinceladas sobre trabajo creativo (productividad de ideas) frente a trabajo máquina (productividad de tareas) -conceptos que nos hacen entender la magnitud de la transformación del tipo de 'organización máquina' -modelo de organización imperante y de la relación organización-trabajo que se requieren para que sea posible expandir el potencial del conocimiento como factor masivo de producción, y reflexionaré sobre lo que mueve a ser creativo.

En la primera parte de este artículo hacía hincapié en la ineficacia de basar la gestión del conocimiento en enfoques técnicoestructurales y defendía la rotunda supremacía de enfoques persona; ya que el conocimiento permite manejarnos en entornos en continuo cambio -para lo que lo estructurado y planificado sirve de bien poco-; ya que el conocimiento está estrechamente relacionado con la creatividad que es inmanente a las personas; ya que lo realmente importante reside en cómo desplegar el potencial creativo de las personas y grupos en aras a crear y manejar conocimiento que aporte valor a la organización. Continúo profundizando en estas reflexiones...

\section{Conocimiento y creatividad}

Crear conocimiento consiste en llegar a una nueva relación entre conocimientos previos que abre nuevas vías de solución o exploración. Esta 'nueva relación' es el producto de la creatividad, de la imaginación. Crear conocimiento útil va a depender de los factores siguientes: base previa de conocimiento, comprensión de los problemas en su extensión y creatividad (potencia y despliegue). Los elementos impulsores de estos tres factores son: el aprendizaje continuo, la comunicación 
y ambientes que propicien la creatividad. A mayor conocimiento previo, aumenta la probabilidad de generar ideas útiles y aportadoras de valor. Así, son elementos muy importantes la formación continua y el estado de alerta a todo lo que ocurra en el exterior, tanto como el aprendizaje de las propias acciones realizadas en el seno de la organización. Dado que el conocimiento se encuentra en cada una de las mentes de las personas que integran la organización, la comunicación es el lubrificante que puede hacer funcionar una red de conocimiento en expansión.

Comprender los problemas en su mayor extensión es imprescindible para que las soluciones que vayan definiéndose aporten valor. Compartir interpretaciones, poner en común, comunicar, aprender de otros es crucial.

La creatividad consiste en encontrar una nueva relación entre elementos conocidos. Algún día alguien conocedor del concepto 'volar' y del concepto 'elefante', los juntó en un 'elefante volador', creando una nueva identidad, aunque limitada a nuestra imaginación. En ese acto creativo puede que hubieran influido otros conceptos de complejidad superior -como la leyenda mitológica de Pegaso- conocidos por el creador. Continuamente se están creando nuevos conceptos, nuevas ideas. Todo nuestro conocimiento es consecuencia de una secuencia de creaciones que se han ido produciendo y expandiendo a lo largo de los siglos. El lenguaje es un buen ejemplo. El conocimiento científico es también una sucesión de modelos teóricos construidos desde la creatividad sobre el conocimiento previo ${ }^{3}$. Sólo se puede generar conocimiento, impres-cindible para innovar, desde la creatividad. Esta frase puede considerarse tautológica. Pero es que además, cuando el conocimiento llega a un nivel alto de complejidad, es imposible manejarlo inteligentemente sin poner en práctica la imaginación. Es obvio que generar nuevo conocimiento requiere creatividad, lo que no se oye tan a menudo - pero resulta igual de obvio a nada que pensemos sobre cualquier ejemplo práctico -es que optimizar su manejo también la requiere. Así, la gestión del cono-cimiento guarda estrechísima relación con el desarrollo de una multitud creativa.

\section{Conocimiento y creatividad: desarrollo de multitudes creativas}

Por todo esto, una organización enfocada hacia las personas es la única manera de llegar a organizaciones basadas en el conocimiento -que en la mayoría de los casos y cada vez más se podría generalizar a su identificación con organizaciones competitivas-, utilizando el enfoque dato como mero elemento que puede ampliar la base de información de la que nutrir el conocimiento.

Distintas actividades que generan valor en una organización pueden ser sistematizadas para mejorar su eficiencia. Con la información pasa lo mismo. Ahora bien, la información debe ser útil para quien la maneja y debe ser una carga soportable para quien la genera. Deben por tanto ser quienes la manejan y generan quienes conjuntamente establezcan cómo -de hacerlo- se ha de sistematizar. Un enfoque dato extremista cae en errores como implantar sistemas de procesado de información que han funcionado en otro lugar -sin tener en cuenta las especificidades de la organización en cuestión-, evaluar la gestión del conocimiento en función de la información que se maneje y no de la utilidad que se le dé; en definitiva lleva a primar la eficiencia en el manejo de la información sobre su utilidad, sobre su creación de valor. Esto no sería tan grave si no fuera que el manejo de la información supone exclusivamente despilfarro de recursos si no hay utilidad.

\footnotetext{
3 Léase el prólogo y el prefacio de Teodoro Vives: «Espacio y Tiempo»: Equipo Sirius, 2006 para profundizar en esta idea.
} 
Enfoque dato y enfoque persona

La creatividad es una actitud individual que en el seno de la organización ve luz cuando se despliega en la multitud. Se trata de construir un estilo a base de motivación mutua, refuerzo positivo, libertad, permisión de errores, que propicie actitudes que se traduzcan en comportamientos abiertos a lo diferente. En un enfoque estructuralista, sistematizador, las personas son consideradas aberrante-mente unidades homogéneas y su conducta se entiende previsible y controlable. Así se definen las pautas de comportamiento, que incluyen componentes actitudinales, como si se tratara de configurar la pantalla inicio del ordenador. Se comete el gravísimo error de obviar los aspectos subjetivos por lo difícil de su manejo.

Generar conocimiento que pueda ser eficaz y productivo depende por encima de todo de la motivación, para aprender, comprender los problemas de otros y para dar rienda suelta a la creatividad; $y$ de otro aspecto que la afecta: el feedback recibido en anteriores ocasiones, es decir, el refuerzo positivo o negativo recogido de comportamientos anteriores. En este contexto, resulta sugerente el parentesco etimológico de 'motivación' y 'emoción'. Lo que me mueve o me motiva son en definitiva mis sentimientos y emociones. Y éste es el fondo de otro concepto hoy de moda, que no por ello debe desacreditarse: la 'Inteligencia Emocional'. Desarrollar esta idea podría ser el núcleo de otro artículo, que excede al presente, pero me parece interesante dejarlo apuntado. Cuando se trata de impulsar una actitud creativa y comunicativa, lo realmente decisivo es la conjunción de emociones y voluntad que hará actuar de una u otra forma. Cuando se pretende que la persona dé lo mejor de sí, su creatividad, tiene que gozar de libertad, emocionarse con lo que está haciendo, sentir que quiere dar.

\section{Lo que mueve a ser creativo}

Las actitudes, los comportamientos, los estilos, los valores no se pueden 'implantar'. Las personas se comportan según parámetros emergentes de su interior, no impuestos desde fuera. No se puede 'exigir' de alguien que sea creativo, no se le puede 'imponer' que sea imaginativo. Ser creativo es algo que sólo sucede cuando se dan determinadas circunstancias en el interior de las personas, que pueden -eso sí- ser propiciadas por un entorno que conecte con la subjetividad creativa. Por el contrario, otro tipo de ambientes, a pesar de mil proclamas, pueden llegar a abortar toda acción imaginativa. OEn sistemas cerrados se frena la diversidad. Se ha hecho, se hace y se seguirá haciendo. Ahora, cuando la potencia de la organización se quiere erigir desde el motor de la subjetividad que construya sistemas abiertos, dinámicos, en continua expansión, la incompatibilidad de raíz entre implantar comportamientos y actitudes cerradas y promover la diversidad, subjetividad, creatividad de cada cual es clara. Cuando se pretende un comportamiento cerrado, rígido, sistematizado, donde la subjetividad de la persona no tiene valor, sino la reproducción de comportamientos cual máquinas, lo que se persigue es precisamente que la persona inhiba su ser creativo y que siga pautas cerradas. Esto ha sido -y, por desgracia, sigue siendo- muy habitual en los diversos ámbitos interpersonales.

En el contexto actual todo esto nos lleva a una reflexión de gran calado. El trabajo máquina pierde peso a favor del trabajo creativo. Es la propia coyuntura económica la que hace mover la balanza. Por suerte, este movimiento tectónico puede ser un estimulante para humanizar todo tipo de relaciones en la pluralidad de ámbitos interpersonales que podamos imaginar.

La gestión del conocimiento es en definitiva propiciar ambientes, estilos, formas que conduzcan al desarrollo y expansión de multitudes creativas que generen y manejen conocimiento que aporte valor. 